\title{
Is B-type natriuretic peptide (BNP) similarly associated with mortality in patients with and without heart failure?
}

\author{
Norihide Fukushima \\ National Cerebral and Cardiovascular Center, Suita, Osaka, Japan \\ Correspondence to: Norihide Fukushima. National Cerebral and Cardiovascular Center, 5-7-1 Fujishiro-dai, Suita, Osaka 565-8565, Japan. \\ Email: nori@ncvc.go.jp. \\ Comment on: York MK, Gupta DK, Reynolds CF, et al. B-Type Natriuretic Peptide Levels and Mortality in Patients With and Without Heart Failure. \\ J Am Coll Cardiol 2018;71:2079-88.
}

Submitted Aug 17, 2018. Accepted for publication Aug 28, 2018.

doi: $10.21037 / \mathrm{atm} .2018 .08 .46$

View this article at: http://dx.doi.org/10.21037/atm.2018.08.46

The natriuretic peptides (NPs) B-type NP and N-terminal (BNP and NT-proBNP, respectively) are peptides derived from proBNP (1). Originally reported to be useful diagnostic and monitoring tools for heart failure (HF), as recommended by international guidelines (2), plasma concentrations of NPs have been revealed to be strong predictor of increased risk for death in patients with acute coronary syndromes in the short and long-term period (3-6). Although NPs have been shown to be associated with no only survival of patients in a broad spectrum of cardiac or non-cardiac diseases [e.g., sepsis, diabetes mellitus, coronary artery disease, pulmonary embolism and renal dysfunction (7-9)], but also that in the general population (10), some important questions remain unresolved.

The study by York et al. (11) published in Fournal of the American College of Cardiology, reported that BNP was a strong prognostic examination tool for death not only in patients with HF but also in those without HF. Briefly, their study was conducted among 30,487 patients who had a first BNP measurement, from the electronic health record data of the Vanderbilt University Medical Center (Nashville, Tennessee, USA). Among those analyzed patients, $50 \%$ was male, the median age was 63 years, $17 \%$ was black, and $38 \%$ was patients with HF. They analyzed the relationship between first measured serum BNP concentration and 3-year all-cause mortality. In this study, a patient was determined as having $\mathrm{HF}$ if either of the following two criteria was met: first, 2 or more statements of a HF code [code 428.x of the International Classification of Diseases, Ninth Revision (ICD-9)], where at least
1 code was recorded on the day or before first BNP was measured. Second, 1 or more statements of code 428.x and intravenous diuretic treatment on the day or within 90 days after the first BNP was measured. Chronic and new HF diagnoses could be captured by these criteria, respectively. Patients with other complications contributing to dyspnea, such as chronic obstructive pulmonary disease, asthma, pneumonitis, and sepsis, as well as those with both HF were categorized as having HF.

York's study (11) also analyzed the relationship between the first measured BNP and other biological and clinical values and revealed that the first, second and third strongest correlates with plasma BNP concentration were increasing age, lesser body mass index (BMI), and greater left ventricle (LV) mass, respectively in the non-HF group. On the other hand, in patients with HF, increasing age was not the strongest correlate, but the first, second, third and fourth strongest correlates with plasma BNP concentration were greater BMI, lower LV ejection fraction (EF), lower estimated glomerular filtration rate (GFR) and chronic kidney disease, respectively. In both groups, female sex, coronary artery disease, lower eGFR, chronic kidney disease, higher heart rate, increasing age, and greater LV mass were correlated with higher plasma BNP concentration, whereas greater BMI and higher LVEF were correlated with lower plasma BNP concentration. Over 90,898 person-years of follow-up, $53 \%$ and $31 \%$ of patients with and without HF died, respectively. Mortality rates at 5 years in overall, outpatient and acute or hospitalized care patients in patients with $\operatorname{HF}(53.9 \%, 40.1 \%$ and $60.2 \%$, respectively) are higher than those in patients without $\mathrm{HF}$ 
(38.7\%, $22.7 \%$ and $45.3 \%$, respectively). In multivariateadjusted Cox models, in patients without HF, higher BNP was the strongest predictor of death, but in patients with $\mathrm{HF}$, the second strongest one, behind increasing age. Higher heart rate and coronary artery disease were the third and fourth strongest predictor among patients without HF, but among patients with HF, chronic kidney disease and coronary artery disease were the third and fourth strongest predictor. Female sex, diabetes mellitus, lower eGFR, greater BMI, higher heart rate, greater LV mass and lower LVEF were correlated with increased risk for death in both groups. In patients without HF, higher heart rate was significantly associated with first measure plasma BNP concentration and the third strongest predictor of death behind higher plasma BNP concentration and increasing age, probably because greater sympathetic activity which may cause higher heart rate, may contribute increased mortality risk in patients without $\mathrm{HF}$ as well as in patients with coronary artery disease and low LVEF.

Based on the above observations, first measured plasma BNP concentration was the strongest predictor of death in patients without HF, even when compared with well-known risk factors such as increasing age, chronic kidney disease, lower eGFR, diabetes, vital signs, greater LV mass, and lower LVEF. This finding is similar to those of communitybased cohorts study reported by Wang et al. (10) in which largely healthy subjects were analyzed. However, the reason why higher BNP may be such a strong predictor for death is incompletely elucidated.

York's study (11) did not clarify the cause of increased plasma BNP concentrations, because the analyzed patients could come into all the categories described above. However, most patients classified as not having HF, had subclinical cardiac disease. For example, in patients without HF, $77 \%$ had hypertension, $45 \%$ had coronary artery disease, and greater than $40 \%$ were treated with anti-HF agents and the median LVEF was 55\%. Costello-Boerrigter et al. (12) reported that female sex and increasing age were the strongest independent predictors of higher plasma NTproBNP concentrations in normal subgroups, and that in patients over 45 years of age, both NT-proBNP and BNP detects a LVEF less than and equal to $40 \%$ with good sensitivity and specificity. On the other hand, the study by McKie et al. (13) reported that the use of NPs was not a prognosticator of mortality in healthy normal subgroups, who did not have general clinical cardiological risk factors and cardiovascular abnormalities evaluated by ultrasonic cardiogram, but in those with stages A and B HF as defined by the American College of Cardiology/American Heart Association (ACC/AHA) (14), NT-proBNP was a good prognosticator of mortality and useful for detection and primary prevention of cardiovascular disease. These reports suggested that even modestly increased plasma BNP concentration can be useful to detect and prevent an asymptomatic cardiac disease (e.g., coronary arterial disease, right and left ventricular hypertrophy, ventricular diastolic dysfunction, tachycardia), which has been reported to be correlated with a risk of cardiac events and death. For example, Omland et al. (15) reported that an increased plasma BNP level was a strong prognosticator of mortality and provided additional prognostic information to general risk factors even in patients with stable and asymptomatic coronary artery disease and preserved LV function. From these kinds of results, recent international guidelines for $\mathrm{HF}$ make cutoffs of plasma NP concentration lower to eliminate subclinical HF $(16,17)$.

It has been well known that increased plasma BNP concentration was also correlated with noncardiac diseases. For example, acute and transient BNP elevation was often observed in patients with sepsis, lung infection, reduced eGFR or subarachnoid hemorrhage, probably because these disorders may reflect poor cardiac reserve. Moreover, many chronic non-cardiac disease, such as, chronic obstructive lung disease, chronic kidney disease diabetes mellitus is also correlated with an increasing plasma NTs concentration. As in York's study (11) and other reports described above (10), aging as well as black race is also associated with increasing plasma NP concentration and mortality. Increasing plasma NP concentration can therefore be a strong predictor of death as well as poor outcomes in many kinds acute and chronic disorders.

The York's study also raised additional important questions about how to treat with patients without HF but with increased plasma NP concentration. In York's study (11), the cause of death or medical interventions after measuring BNP were not available in most cases. Although acute and persistent therapeutic interventions to patients can promptly change the value of plasma NP concentration, they described that the time of measurement of plasma BNP concentration were measured played almost no role on predicting prognosis of the patient, because the first measured plasma BNP concentration was the strongest prognosticator for death in patients without HF. This finding is far from usual clinical observations, because optimal monitoring of plasma NPs concentration in both groups is useful to help anticipate the effects of therapeutic 
strategies as well as preventive methods.

In usual clinical settings, plasma BNP concentration is measured when clinical signs/symptoms and examination suggested that a patient has or at least cardiac disease. In York's study, at lower plasma BNP concentration, 3 -year mortality rates according to any given plasma $\mathrm{BNP}$ concentration was greater in patients with $\mathrm{HF}$ than those without $\mathrm{HF}$, but if the first measured plasma BNP concentration was modestly or higher elevated, the risk of mortality in both groups were similar. For example, the 3-year mortality rate at a plasma BNP concentration of $55 \mathrm{pg} / \mathrm{mL}$ in outpatient patients without and with HF was $5.5 \%$ and $8.0 \%$, respectively and in acute and hospitalized patients without and with $\mathrm{HF}$ was $20 \%$ and $21 \%$, respectively. And that of $403 \mathrm{pg} / \mathrm{mL}$ in outpatient patients without and with $\mathrm{HF}$ was $12 \%$ and $15 \%$, respectively and in acute and hospitalized patients without and with $\mathrm{HF}$ was $30 \%$ and $33 \%$, respectively. Therefore, if a plasma BNP concentration increases $>35 \mathrm{pg} / \mathrm{mL}$ in outpatients and $>100 \mathrm{pg} / \mathrm{mL}$ in acute care, a more extensive cardiovascular evaluation with echocardiography and electrocardiography is recommended. Even if these examinations do not show some kinds of cardiac dysfunction, evaluation of the presence of coronary artery disease and measurement other cardiac biomarkers, such as troponin (18) and creatinine kinase isotype $\mathrm{MB}$ and noncardiac biomarkers, such as high sensitivity C-reactive protein (19) and blood sugar are recommended. Indeed, according to the STOPHF (St. Vincent's Screening to Prevent Heart Failure) trial (8), cardiovascular examination and subsequent care for patients with a screened plasma BNP concentration $>50 \mathrm{pg} / \mathrm{mL}$ significantly reduced the rate of the long-term occurrence of asymptomatic LV dysfunction or incident of HF than a control group without BNP screening. The PONTIAC (NTproBNP Selected Prevention of Cardiac Events in a Population of Diabetic Patients Without a History of Cardiac Disease) study (20) also demonstrated that a gradual increase in a dose of Angiotensin II Receptor Blocker, Angiotensin-converting-enzyme inhibitor and beta-adrenaline blockers in patients with high-risk diabetes and increased plasma NP concentration but without known cardiac disease were association with a reduced risk of hospitalization or cardiovascular death after 2 years.

In conclusion, York's study (11) proves that the first measured plasma BNP concentration is the strongest predictive tool for the increased risk of death even in patients without HF, but raises unsolved important question about what should be done to patients with a modestly or higher elevated plasma BNP concentration such as $>35 \mathrm{pg} / \mathrm{mL}$ in outpatients and $>100 \mathrm{pg} / \mathrm{mL}$ in acute care even in patients without HF. By identifying the role of NPs in a prognostic tool of death in patients whose plasma BNP concentration was measured for any purpose, regardless of $\mathrm{HF}$ status and the purpose of measurement of plasma BNP concentration, standardized management for these patients will become defined.

\section{Acknowledgements}

None.

\section{Footnote}

Conflicts of Interest: The author has no conflicts of interest to declare.

\section{References}

1. de Lemos JA, McGuire DK, Drazner MH. B-type natriuretic peptide in cardiovascular disease. Lancet 2003;362:316-22.

2. Maisel AS, Krishnaswamy P, Nowak RM, et al. Rapid measurement of B-type natriuretic peptide in the emergency diagnosis of heart failure. $\mathrm{N}$ Engl J Med 2002;347:161-7.

3. Omland T, Aakvaag A, Bonarjee VV, et al. Plasma brain natriuretic peptide as an indicator of left ventricular systolic function and long-term survival after acute myocardial infarction. Comparison with plasma atrial natriuretic peptide and $\mathrm{N}$-terminal proatrial natriuretic peptide. Circulation 1996;93:1963-9.

4. de Lemos JA, Morrow DA, Bentley JH, et al. The prognostic value of B-type natriuretic peptide in patients with acute coronary syndromes. N Engl J Med 2001;345:1014-21.

5. Omland T, Persson A, Ng L, et al. N-terminal pro-Btype natriuretic peptide and long-term mortality in acute coronary syndromes. Circulation 2002;106:2913-8.

6. Morrow DA, de Lemos JA, Blazing MA, et al. Prognostic value of serial B-type natriuretic peptide testing during follow-up of patients with unstable coronary artery disease. JAMA 2005;294:2866-71.

7. Wolsk E, Claggett B, Pfeffer MA, et al. Role of B-type natriuretic peptide and $\mathrm{N}$-terminal prohormone BNP as predictors of cardiovascular morbidity and mortality in patients with a recent coronary event and type 2 
diabetes mellitus. J Am Heart Assoc 2017;6. doi: 10.1161/ JAHA.116.004743.

8. Ledwidge M, Gallagher J, Conlon C, et al. Natriuretic peptide-based screening and collaborative care for heart failure: the STOP-HF randomized trial. JAMA 2013;310:66-74.

9. McGrady M, Reid CM, Shiel L, et al. N-terminal B-type natriuretic peptide and the association with left ventricular diastolic function in a population at high risk of incident heart failure: results of the SCReening Evaluationof the Evolution of New Heart Failure Study (SCREEN-HF). Eur J Heart Fail 2013;15:573-80.

10. Wang TJ, Larson MG, Levy D, et al. Plasma natriuretic peptide levels and the risk of cardiovascular events and death. N Engl J Med 2004;350:655-63.

11. York MK, Gupta DK, Reynolds CF, et al. B-Type Natriuretic Peptide Levels and Mortality in Patients With and Without Heart Failure. J Am Coll Cardiol 2018;71:2079-88.

12. Costello-Boerrigter LC, Boerrigter G, Redfield MM, et al. Amino-Terminal Pro-B-Type Natriuretic Peptide and B-Type Natriuretic Peptide in the General Community Determinants and Detection of Left Ventricular Dysfunction. J Am Coll Cardiol 2006;47:345-53.

13. McKie PM, Cataliotti A, Lahr BD, et al. The prognostic value of $\mathrm{N}$-terminal Pro-B-type natriuretic peptide for death and cardiovascular events in healthy normal and stage $\mathrm{A} / \mathrm{B}$ heart failure subjects. J Am Coll Cardiol 2010;55:2140-7.

14. Ammar KA, Jacobsen SJ, Mahoney DW, et al. Prevalence and prognostic significance of heart failure stages: application of the American College of Cardiology/ American Heart Association heart failure staging criteria

Cite this article as: Fukushima N. Is B-type natriuretic peptide (BNP) similarly associated with mortality in patients with and without heart failure? Ann Transl Med 2018;6(Suppl 1):S9. doi: 10.21037/atm.2018.08.46 in the community. Circulation 2007;115:1563-70.

15. Omland T, Sabatine MS, Jablonski KA, et al. Prognostic Value of B-Type Natriuretic Peptides in Patients With Stable Coronary Artery Disease: The PEACE Trial. J Am Coll Cardiol 2007;50:205-14.

16. Ponikowski P, Voors AA, Anker SD, et al. 2016 ESC guidelines for the diagnosis and treatment of acute and chronic heart failure: The Task Force for the Diagnosis and Treatment of Acute and Chronic Heart Failure of the European Society of Cardiology (ESC) developed with the special contribution of the Heart Failure Association (HFA) of the ESC. Eur Heart J 2016;37:2129-200.

17. Yancy CW, Jessup M, Bozkurt B, et al. 2017 ACC/ AHA/HFSA focused update of the 2013 ACCF/AHA guideline for the management of heart failure: a report of the American College of Cardiology/American Heart Association Task Force on Clinical Practice Guidelines and the Heart Failure Society of America. J Am Coll Cardiol 2017;70:776-803.

18. McKie PM, AbouEzzeddine OF, Scott CG, et al. Highsensitivity troponin I and amino-terminal pro-B-type natriuretic peptide predict heart failure and mortality in the general population. Clin Chem 2014;60:1225-33.

19. Sigurdardottir FD, Lyngbakken MN, Holmen OL, et al. Relative Prognostic Value of Cardiac Troponin I and C-Reactive Protein in the General Population (from the Nord-Trøndelag Health [HUNT] Study). Am J Cardiol 2018;121:949-55.

20. Huelsmann M, Neuhold S, Resl M, et al. PONTIAC (NT-proBNP Selected Prevention of Cardiac Events in a Population of Diabetic Patients Without a History of Cardiac Disease): a prospective randomized controlled trial. J Am Coll Cardiol 2013;62:1365-72. 